\title{
Image Deblurring Using a Perturbation-Based Regularization Approach
}

\author{
Abdulrahman M. Alanazi ${ }^{1,2}$, Tarig Ballal ${ }^{1}$, Mudassir Masood ${ }^{3}$, and Tareq Y. Al-Naffouri ${ }^{1}$ \\ ${ }^{1}$ King Abdullah University of Science and Technology (KAUST), Thuwal, Saudi Arabia. \\ ${ }^{2}$ King Saud University (KSU), Riyadh, Saudi Arabia. \\ ${ }^{3}$ King Fahd University of Petroleum and Minerals (KFUPM), Dhahran, Saudi Arabia.
}

\begin{abstract}
The image restoration problem deals with images in which information has been degraded by blur or noise. In this work, we present a new method for image deblurring by solving a regularized linear least-squares problem. In the proposed method, a synthetic perturbation matrix with a bounded norm is forced into the discrete ill-conditioned model matrix. This perturbation is added to enhance the singular-value structure of the matrix and hence to provide an improved solution. A method is proposed to find a near-optimal value of the regularization parameter for the proposed approach. To reduce the computational complexity, we present a technique based on the bootstrapping method to estimate the regularization parameter for both low and high-resolution images. Experimental results on the image deblurring problem are presented. Comparisons are made with three benchmark methods and the results demonstrate that the proposed method clearly outperforms the other methods in terms of both the output PSNR and SSIM values.
\end{abstract}

Index Terms-Bootstrapping, bounded perturbation regularization, image deblurring, linear least-squares problems, Tikhonov regularization.

\section{INTRODUCTION}

Image restoration from blurry and noisy observations has received great interest over the years. Often, the problem is reduced to an ill-conditioned linear least-squares problem of a very large dimension. One approach to solving the image deblurring problem is to solve a regularized version of the problem by adding to the least-squares optimization criterion a supplementary penalizing term, that accounts for additional desirable properties of the solution. One possible choice for the additional term is the total variation seminorm [1-3]. By attempting to minimize the total variation, the restored image tends to be smooth. Several image restoration algorithms that consider total variation regularization have been proposed, for example [3] and [4]. It is often advantageous to use the sparsity of the high-frequency components of the image (such as edges), and this can be done either using wavelet methods [5] or total variation regularization such as in [1], [2]. A particular class of techniques to handle these kinds of regularizers consists of iterative shrinkage/thresholding algorithms as proposed in [6].

A popular regularization approach is the $\ell_{2}$ or Tikhonov regularization, which has a closed-form solution. The choice of the regularization parameter for Tikhonov-based regularization is an active area of research. In [7], Reeves attempted to solve the image deblurring problem by solving the regularized least-squares problem using the so-called generalized cross validation (GCV) method to find the regularization parameter. A different regularization method, named the L-curve regularization for the same type of problems is proposed in [8]. The algorithm is illustrated on a number of test problems, including an image deblurring application.

Image restoration requires computationally intensive inversion of very large matrices. Matrix inversion is not required only (once) for restoring the image but also is repetitively needed to compute the regularization parameter. In the image deblurring problem, the blur matrix is constructed from the point spread function (PSF). This matrix is structured based on the boundary condition (BC) assumption which describes the behavior of the image outside the scene. For computational convenience, a periodic BC is often assumed which results in a circulant blur matrix. Hence, the computation is done efficiently using fast Fourier transforms (FFT). However, the periodic $\mathrm{BC}$ assumption makes sense only when the image is symmetric. Also, zero BC is usually assumed in astronomical images where the image has a black background, and the blur matrix in this case is Toeplitz. Thus, direct filtering type methods cannot be used as efficiently as with circulant matrices [9]. A more realistic assumption is a synthetic boundary condition (SBC) which sensibly extends the pixels across the boundary [10]. However, in this case, the structure of the blur matrix does not allow for efficient implementation; so the matrix inversion is computationally complex.

In this paper, we propose using a new approach for image deblurring. The proposed method is based on allowing perturbation into the model matrix and solving the resultant problem. We focus on the case where the blur matrix is constructed; namely, with SBC. To reduce the computational complexity, we propose a technique based on the bootstrap principle. In the following section, we will present the derivation of the proposed method. Following the derivation, we will show how to reduce the computational complexity in Section III. Then, image deblurring results are presented in Section IV.

\section{THE PROPOSED REGULARIZATION APPROACH}

In image deblurring, we seek to restore the image using a mathematical model of the form

$$
\mathbf{b}=\mathbf{A x}+\mathbf{w},
$$


where $\mathbf{b} \in \mathbb{R}^{M}$ is the observed image of length $M=m \times n$, $\mathbf{x} \in \mathbb{R}^{N}$ is the original image, $\mathbf{A} \in \mathbb{R}^{M \times N}$ is a linear operator that represents the blur matrix, and $\mathbf{w} \in \mathbb{R}^{M}$ is an additive white Gaussian noise with zero mean and standard deviation $\sigma$. Similar to [11] and [12], we perturb the mathematical model (1) to

$$
\mathbf{b} \approx(\mathbf{A}+\boldsymbol{\Delta}) \mathbf{x}+\mathbf{w},
$$

where $\boldsymbol{\Delta} \in \mathbb{R}^{M \times N}$ is an unknown perturbation matrix. The reason for adding the perturbation is to improve the structure of the singular values of the matrix A. By deciding to go from (1) to (2), we choose to tradeoff the model accuracy for model robustness. We envision that, for a certain perturbation $\boldsymbol{\Delta}$, the gain in robustness against blur and noise overweighs the loss in model accuracy. It is obvious that the perturbation should somehow be bounded-adding too much perturbation to the model can destroy the model fidelity. Therefore, we bound the 2-induced norm of the matrix $\Delta$ by a positive amount $\zeta$, i.e., $\|\boldsymbol{\Delta}\|_{2} \leq \zeta$. This bound is generally not known and is a key subject of the proposed approach. To proceed further, we will assume, for the time being, that $\zeta$ is a constant whose value is known. Later we will make up for the lack of knowledge of the bound constant $\zeta$. To find an estimate of $\mathbf{x}$, we pursue the following min-max approach,

$$
\min _{\hat{\mathbf{x}}} \max _{\boldsymbol{\Delta}}|| \mathbf{b}-(\mathbf{A}+\boldsymbol{\Delta}) \hat{\mathbf{x}} \|_{2}, \quad \text { s. t. }\|\boldsymbol{\Delta}\|_{2} \leq \zeta .
$$

The rationale behind (3) is that we seek an estimate of $\mathbf{x}$ which minimizes the maximum residual error over all possible bounded perturbations $\Delta$. It can be shown that solving the min-max problem (3) is equivalent to solving the unconstrained optimization problem [13]

$$
\min _{\hat{\mathbf{x}}}|| \mathbf{b}-\mathbf{A} \hat{\mathbf{x}}\left\|_{2}+\zeta\right\| \hat{\mathbf{x}} \|_{2} .
$$

The cost function in (4) is convex and hence the minimizer can be obtained by differentiating the function and equating to zero. Thus, the solution to (4) is given by

$$
\hat{\mathbf{x}}_{\mathrm{RLS}}=\left(\mathbf{A}^{T} \mathbf{A}+\gamma \mathbf{I}\right)^{-1} \mathbf{A}^{T} \mathbf{b},
$$

where $\gamma$ is given by

$$
\gamma=\frac{\zeta\|\mathbf{b}-\mathbf{A} \hat{\mathbf{x}}\|_{2}}{\|\hat{\mathbf{x}}\|_{2}} .
$$

Substituting the singular-value decomposition (SVD) $\mathbf{A}=\mathbf{U} \boldsymbol{\Sigma} \mathbf{V}^{\mathbf{T}}$ in (5), we obtain

$$
\hat{\mathbf{x}}_{\mathrm{RLS}}=\mathbf{V}\left(\boldsymbol{\Sigma}^{2}+\gamma \mathbf{I}\right)^{-1} \boldsymbol{\Sigma} \mathbf{U}^{T} \mathbf{b} .
$$

Substituting (7) and $\mathbf{A}=\mathbf{U} \boldsymbol{\Sigma} \mathbf{V}^{\mathbf{T}}$ in (6) and manipulating, results in

$$
\mathbf{b}^{T} \mathbf{U}\left(\boldsymbol{\Sigma}^{2}-\zeta^{2} \mathbf{I}\right)\left(\boldsymbol{\Sigma}^{2}+\gamma \mathbf{I}\right)^{-2} \mathbf{U}^{T} \mathbf{b}=0 .
$$

It has been shown in [11] that a near-optimal choice of the parameter $\zeta$, is given by

$$
\zeta^{2}=\frac{\operatorname{tr}\left[\boldsymbol{\Sigma}^{2}\left(\boldsymbol{\Sigma}^{2}+\gamma \mathbf{I}\right)^{-1}\right]}{\operatorname{tr}\left[\left(\boldsymbol{\Sigma}^{2}+\gamma \mathbf{I}\right)^{-1}\right]},
$$

where $\operatorname{tr}[\cdot]$ denotes the trace of a matrix. By inserting (9) in (8) and manipulating, we reach at the following equation

$$
\begin{aligned}
f(\gamma) & =\operatorname{tr}\left[\left(\boldsymbol{\Sigma}^{2}+\gamma \mathbf{I}\right)^{-1}\right] \operatorname{tr}\left[\left(\boldsymbol{\Sigma}^{2}+\gamma \mathbf{I}\right)^{-1} \mathbf{U}^{T} \mathbf{b} \mathbf{b}^{T} \mathbf{U}\right] \\
& -N \operatorname{tr}\left[\left(\boldsymbol{\Sigma}^{2}+\gamma \mathbf{I}\right)^{-2} \mathbf{U}^{T} \mathbf{b} \mathbf{b}^{T} \mathbf{U}\right]=0,
\end{aligned}
$$

where $N$ is the length of the vector $\mathbf{x}$. Equation (10) relates the unknown regularization parameter to the known parameters of the model (1). Now, we need to find a positive root of $f(\gamma)$. Note that since $f(\gamma)$ is differentiable and its derivative $f^{\prime}(\gamma)$ can be easily obtained, one can use Newton's method [14] to obtain the root. The method can be applied by setting an initial value $\gamma^{k=0}$, where $k$ denotes the iteration number. Then, we perform the following iterations:

$$
\gamma^{k+1}=\gamma^{k}-\frac{f\left(\gamma^{k}\right)}{f^{\prime}\left(\gamma^{k}\right)}, \quad k=0,1,2, \cdots,
$$

and terminate when $\left|f\left(\gamma^{k+1}\right)\right|<\epsilon$, where $\epsilon$ is a sufficiently small positive value and $|$.$| denotes the absolute value. It can be$ mathematically shown that Equation 11 will always converge to a unique positive root if a certain condition that depends on the model matrix and noise levels is satisfied. Experimental tests have shown that this condition is always satisfied in practical noise levels. The details of this condition are omitted for the sake of conciseness. However, the proof of convergence is provided in [13].

\section{COMPLEXITy REDUCTION OF THE PROPOSED METHOD}

The computational complexity of the proposed approach is dominated by the complexity of the computation of the SVD of matrix A. In Section II, we relied on SVD to facilitate the computations of the matrix inversions required for computing the regularization parameter. Since we do not assume any structure of A, computing the SVD can be computationally demanding especially for large dimensions since its complexity is of order $\mathcal{O}\left(M^{2} N+N^{3}\right)$. To reduce the complexity of the proposed algorithm, we utilize the powerful technique of bootstrapping [15]. We sub-sample the image to build $K$ models of reduced dimensions and then compute the regularization parameter for each sub-sample independently. In the sequel, we will show that substantial reduction in computational complexity can be achieved by sub-sampling the observed image to a significantly smaller dimension. Subsampling the observed image in model (1) results in the model

$$
\tilde{\mathbf{b}}_{i}=\tilde{\mathbf{A}}_{i} \tilde{\mathbf{x}}_{i}+\tilde{\mathbf{w}}_{i}, \quad \forall i \in\{1,2, \cdots, K\},
$$

where $(\tilde{\cdot})_{i}$ is the sub-sampled version of the corresponding vector/matrix in (1) and $\tilde{\mathbf{b}}_{i} \in \mathbb{R}^{P}(P<M)$ of length $P=s \times$ $r, \tilde{\mathbf{x}}_{i} \in \mathbb{R}^{Q}(Q<N), \tilde{\mathbf{A}}_{i} \in \mathbb{R}^{P \times Q}$, and $\mathbf{w} \in \mathbb{R}^{P} . K$ denotes the number of sub-sampled images $\tilde{\mathbf{b}}_{i}$ that we extract from the observed image $\mathbf{b}$. By applying the SVD on the blur matrix $\tilde{\mathbf{A}}_{i}$ of the sub-sampled image, we obtain $\tilde{\mathbf{A}}_{i}=\tilde{\mathbf{U}}_{i} \tilde{\boldsymbol{\Sigma}}_{i} \tilde{\mathbf{V}}_{i}^{T}$. It is clear that the SVD computation of $\tilde{\mathbf{A}}_{i}$ is less computationally demanding than the SVD of the full blur matrix $\mathbf{A}$ since its 


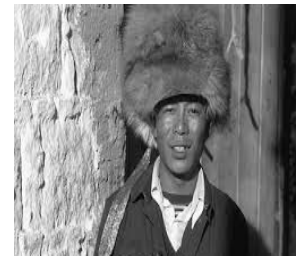

Man $(275 \times 183)$

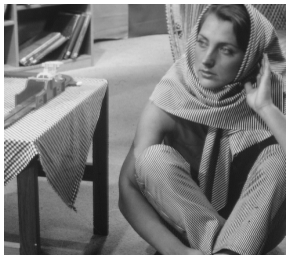

Barbara $(512 \times 512)$

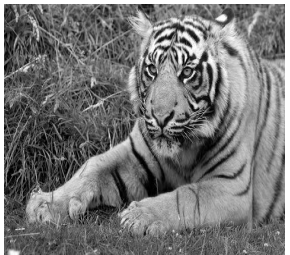

Tiger $(500 \times 330)$

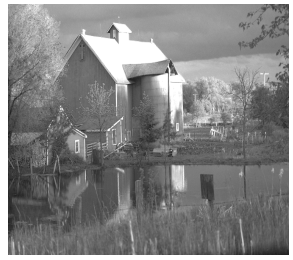

Barn $(768 \times 512)$
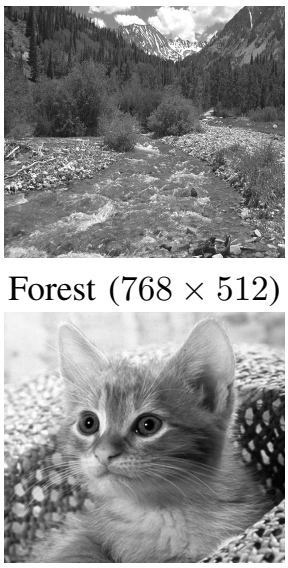

Cat $(350 \times 350)$

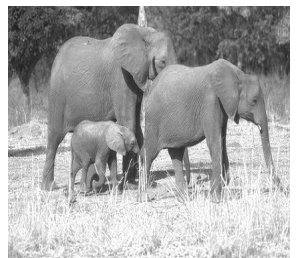

Elephant $(450 \times 301)$

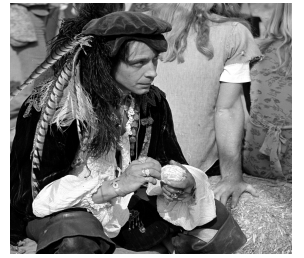

Magician $(1024 \times 1024)$

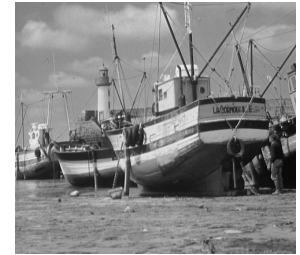

Ships $(512 \times 512)$

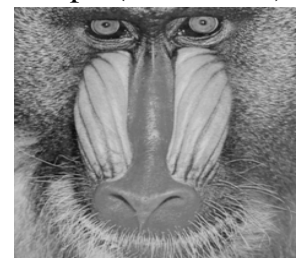

Mandrill $(256 \times 256)$

Figure 1: Original test images with different dimensions.

complexity is of the order $\mathcal{O}\left(P^{2} Q+Q^{3}\right)$. By choosing $P$ and $Q$ to be sufficiently small compared to $M$ and $\mathrm{N}$, significant reduction in computational complexity can be achieved. For the model in Equation (12), (10) becomes

$$
\begin{aligned}
f\left(\tilde{\gamma}_{i}\right) & =\operatorname{tr}\left[\left(\tilde{\boldsymbol{\Sigma}}_{i}^{2}+\tilde{\gamma}_{i} \mathbf{I}\right)^{-1}\right] \operatorname{tr}\left[\left(\tilde{\boldsymbol{\Sigma}}_{i}^{2}+\tilde{\gamma}_{i} \mathbf{I}\right)^{-1} \tilde{\mathbf{U}}_{i}^{T} \tilde{\mathbf{b}}_{i} \tilde{\mathbf{b}}_{i}^{T} \tilde{\mathbf{U}}_{i}\right] \\
& -Q \operatorname{tr}\left[\left(\tilde{\boldsymbol{\Sigma}}_{i}^{2}+\tilde{\gamma}_{i} \mathbf{I}\right)^{-2} \tilde{\mathbf{U}}_{i}^{T} \tilde{\mathbf{b}}_{i} \tilde{\mathbf{b}}_{i}^{T} \tilde{\mathbf{U}}_{i}\right] \\
& =0
\end{aligned}
$$

where $\tilde{\gamma}_{i}$ is the regularization parameter estimate obtained from the sub-sampled image $\tilde{\mathbf{b}}_{i}$. Note that (10) and (13) do not require any complex computations since the matrix inversion is done only for diagonal matrices.

\section{A. Sub-sampling methodology and estimate selection}

One can generate a large number of sub-sampled images by randomly drawing samples from the image. For bootstrapping to yield good results, a sufficient number of sub-samples $(K)$ is needed [15]. A sub-sampled image $\tilde{\mathbf{b}}_{i}$ with size $P$ is obtained by first generating a random set of indices $\mathbf{y}_{i} \subset\{1, M\}$ with cardinality $P$. The sub-sampled image is given by $\tilde{\mathbf{b}}_{i}=\mathbf{b}\left(\mathbf{y}_{i}\right)$, i.e., $\tilde{\mathbf{b}}_{i}$ contains the samples of $\mathbf{b}$ at indices $\mathbf{y}_{i}$. We repeat this for $i=1,2, \cdots, K$, each time using a different set of random indices $\mathbf{y}_{i}$. Note that the size of the sub-sampled image needs to be sufficiently large such that the sub-sampled images are good representation of the full-image. The bootstrap estimate $\tilde{\gamma}$ is obtained by computing the median of $\tilde{\gamma}_{i}$ as follows:

$$
\tilde{\gamma}=\operatorname{median}\left(\tilde{\gamma}_{i}\right), \quad \forall i \in\{1,2, \cdots, K\} .
$$

Median has been shown to be a good estimator in bootstrap theory [15]. Table I demonstrates the estimated regularization parameter $\gamma$ for the full-image, and $\tilde{\gamma}$ which is the bootstrap estimate obtained from $K$ sub-samples each with cardinality $P$ (which is equivalent to $s \times r$ in two-dimensional). In both cases, we show the elapsed time (ET) for computing the regularization parameter estimate and the total construction time (TCT), which is the ET in addition to the deblurring step. For deblurring, we use an efficient iterative method called LSQR [16]. The computations were performed using MATLAB R2016a on Intel(R) Core(TM) i7 CPU E5-2603 $0 @ 1.80$ GHz, 32.00 GB

\begin{tabular}{|c|c|c|c|c|c|c|c|c|}
\hline \multirow{2}{*}{ Image size } & \multicolumn{3}{|c|}{ Full image } & \multicolumn{5}{|c|}{ Sub-sampled image } \\
\hline & $\gamma$ & ET & TCT & $K$ & $s$ & $\tilde{\gamma}$ & ET & TCT \\
\hline $64 \times 64$ & 0.0042 & 35.2 & 37.6 & 8 & 256 & 0.0046 & 4.3 & 5.7 \\
\hline $128 \times 128$ & 0.0067 & 2477 & 2484 & 8 & 256 & 0.0065 & 4.1 & 6.5 \\
\hline $275 \times 183$ & 0.0132 & 10864 & 10875 & 12 & 576 & 0.0134 & 6.6 & 10.4 \\
\hline $256 \times 256$ & 0.0092 & 28403 & 28417 & 12 & 576 & 0.0089 & 7.3 & 13.1 \\
\hline $450 \times 301$ & 0.0771 & 75622 & 75658 & 12 & 576 & 0.0779 & 5.7 & 16.7 \\
\hline $1024 \times 1024$ & \multicolumn{3}{|c|}{ Out-of-memory } & 16 & 1024 & 0.0457 & 9.7 & 27.3 \\
\hline
\end{tabular}
RAM. It is obvious from Table I that using bootstrap produces

Table I: Computation time (in seconds) using the full-image and the sub-sampled image.

nearly the same value of the regularization parameter in a substantially shorter computational time. Using bootstrap, the proposed method is capable of deblurring large-scale images in an efficient manner. The proposed bootstrapping algorithm to find the regularization parameter and restore the image is sketched as Algorithm 1.

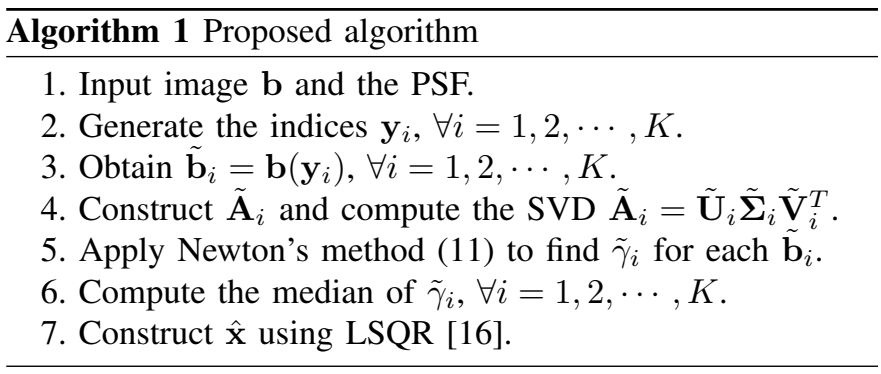

\section{EXPERIMENTAL RESULTS}

The proposed method was tested using various grayscale standard and natural images with low and high-resolutions shown in Figure 1. The natural images are adopted from [17] 
and Kodak website (http://r0k.us/graphics/kodak/). Five real world camera shake kernels adopted from [18] were used to generate the blurry images, and a SBC is assumed. Gaussian white noise with zero mean and three different standard deviation values $(\sigma=0.0005,0.005$ and 0.05$)$ was added to the blurred images. The following benchmark methods were used for comparison: The Augmented Lagrangian Method for Total Variation (ALMTV) [4], the General Framework for Regularized, Similarity-Based Image Restoration (GFRSB) [19], and the Generalized Iterated Shrinkage Algorithm for Non-convex Sparse Coding (GISA) [18]. All the parameters of the benchmark methods were set as suggested by the authors. To give quantitative performance measures, we computed the peak signal-to-noise ratio (PSNR) [20], and the structural similarity (SSIM) index [21]. The plots shown in Figure 2 demonstrate the variation of the output PSNR and SSIM versus the SNR for our proposed method and the benchmark methods on Barbara image. It is clear that the proposed algorithm outperforms the other three methods across the considered SNR range. Figure 3 and Figure 4 illustrate the performance of
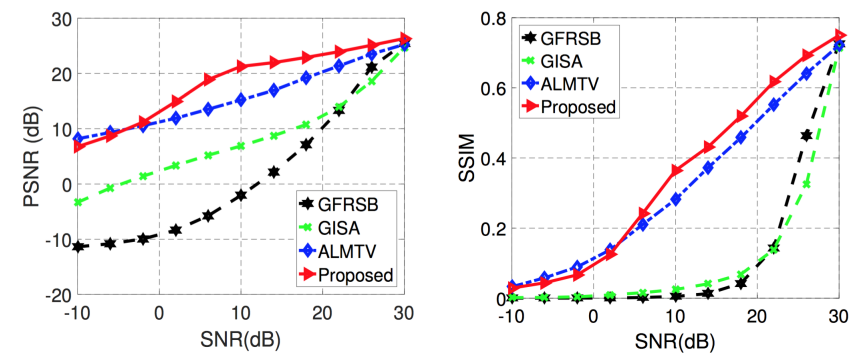

Figure 2: PSNR and SSIM versus SNR comparison over Barbara image.

the tested methods when applied to the Mandrill and Elephant images, respectively. The images were severely blurred with a motion blur kernel, and contaminated with noise level $\sigma=0.005$. The Mandrill image shown in Figure 3 has some fine visual details that are difficult to preserve. The hair texture is everywhere in the image which increases the difficulty of deblurring. In spite of the difficulty to recover these tiny textures, one could still notice that the restored image by the proposed method is much sharper than those obtained from the other algorithms. The image in Figure 4 is a typical natural image with grass and also some wrinkles on the elephant skin. From the results, we can observe that our method is capable of preserving these details better than the other methods. More experimental results are available in [13]. From these results, it is evident that the proposed approach produces images with improved visual quality compared to ALMTV, GFRSB and GISA. Table II and Table III show the average PSNR and SSIM values for the deblurred images obtained from the various methods. The average PSNR and SSIM values were computed for each image over the five blur kernels as suggested by [17]. From the tables, it can be seen that our proposed method clearly outperforms all the other algorithms in terms of the PSNR and SSIM metrics.

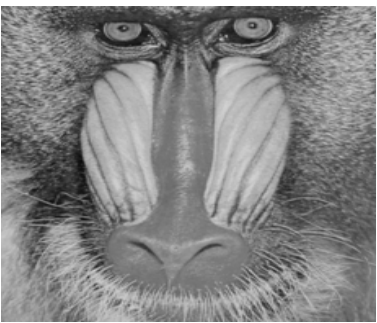

Original image.
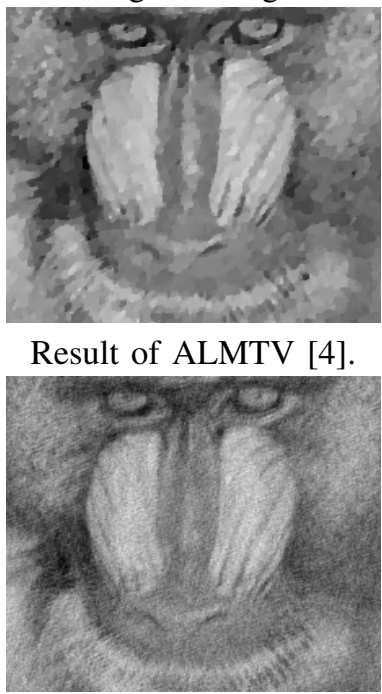

Result of GFRSB [18].

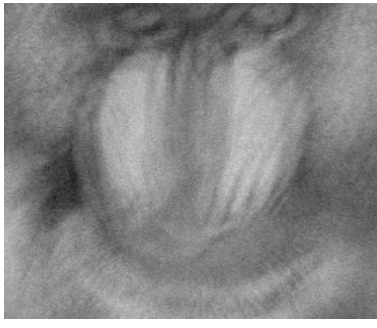

Blurred and noisy image.

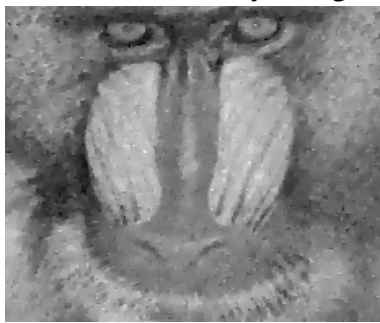

Result of GISA [19].

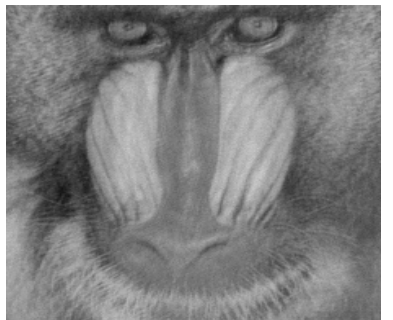

Proposed method.
Figure 3: Performance of the various methods on the Mandrill image.

\section{CONCLUSION}

In this paper, we proposed a new method for the restoration of blurred-and-noisy images. The technique is based on solving the regularized least-squares problem and applying bootstrapping to reduce the computational complexity. Numerical results demonstrate that the proposed algorithm outperforms all the tested benchmark methods in terms of the output PSNR, structural similarity as well as the visual quality of the deblurred images.

\section{ACKNOWLEDGMENT}

This publication is based upon work supported by the King Abdullah University of Science and Technology (KAUST) Office of Sponsored Research (OSR) under Award No. OSR2016-KKI-2899.

\section{REFERENCES}

[1] L. I. Rudin, S. Osher, and E. Fatemi, "Nonlinear total variation based noise removal algorithms," Physica D: Nonlinear Phenomena, vol. 60, no. 1, pp. 259-268, 1992

[2] J. P. Oliveira, J. M. Bioucas-Dias, and M. A. Figueiredo, "Adaptive total variation image deblurring: a majorization-minimization approach," Signal Processing, vol. 89, no. 9, pp. 1683-1693, 2009.

[3] A. Beck and M. Teboulle, "Fast gradient-based algorithms for constrained total variation image denoising and deblurring problems," Image Processing, IEEE Transactions on, vol. 18, no. 11, pp. 2419-2434, 2009. 
Table II: Average PSNR values (in $\mathrm{dB}$ ) over the five blur kernels for the proposed and benchmark methods.

\begin{tabular}{ccccccccccc}
\hline Method & Man & Tiger & Forest & Elephant & Ships & Barbara & Barn & Cat & Magician & Mandrill \\
\hline ALMTV & 15.71 & 16.54 & 14.98 & 18.08 & 16.68 & 15.86 & 18.53 & 17.63 & 12.78 & 16.97 \\
GISA & 13.58 & 15.88 & 15.25 & 17.31 & 15.88 & 15.12 & 17.93 & 17.54 & 12.25 & 17.12 \\
GFRSB & 12.17 & 14.05 & 14.12 & 13.97 & 12.31 & 14.77 & 15.06 & 16.05 & 11.06 & 15.34 \\
Proposed & $\mathbf{1 6 . 1 1}$ & $\mathbf{1 7 . 0 3}$ & $\mathbf{1 5 . 2 8}$ & $\mathbf{1 9 . 4 2}$ & $\mathbf{1 7 . 3 4}$ & $\mathbf{1 5 . 9 8}$ & $\mathbf{1 8 . 7 2}$ & $\mathbf{1 7 . 8 6}$ & $\mathbf{1 3 . 2 4}$ & $\mathbf{2 1 . 2 2}$ \\
\hline
\end{tabular}

Table III: Average SSIM values over the five blur kernels for the proposed and benchmark methods.

\begin{tabular}{ccccccccccc}
\hline Method & Man & Tiger & Forest & Elephant & Ships & Barbara & Barn & Cat & Magician & Mandrill \\
\hline ALMTV & 0.45 & 0.60 & 0.52 & 0.66 & 0.52 & 0.53 & 0.49 & 0.54 & 0.39 & 0.59 \\
GISA & 0.41 & 0.52 & 0.57 & 0.59 & 0.56 & 0.55 & 0.53 & 0.58 & 0.41 & 0.62 \\
GFRSB & 0.23 & 0.45 & 0.43 & 0.52 & 0.47 & 0.45 & 0.42 & 0.46 & 0.32 & 0.55 \\
Proposed & $\mathbf{0 . 5 4}$ & $\mathbf{0 . 6 6}$ & $\mathbf{0 . 5 9}$ & $\mathbf{0 . 7 1}$ & $\mathbf{0 . 6 1}$ & $\mathbf{0 . 5 9}$ & $\mathbf{0 . 5 6}$ & $\mathbf{0 . 6 0}$ & $\mathbf{0 . 4 3}$ & $\mathbf{0 . 7 2}$ \\
\hline
\end{tabular}

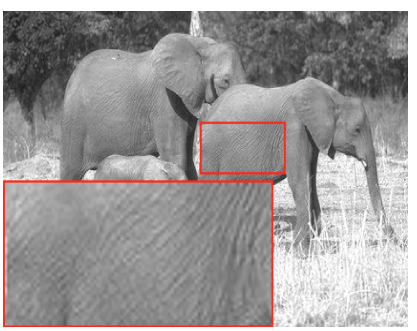

Zoom-in original image.

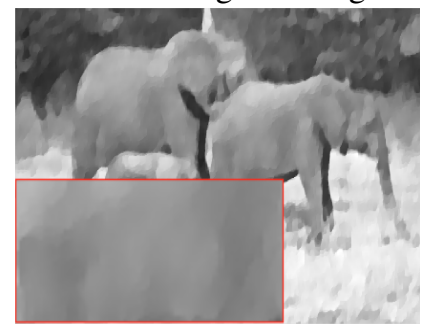

Result of ALMTV [4].

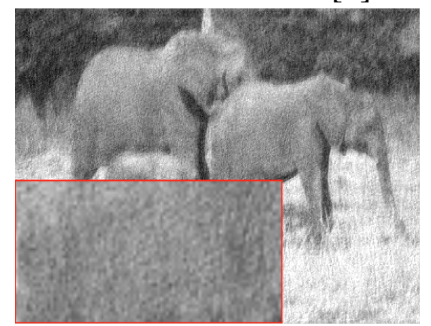

Result of GFRSB [18].

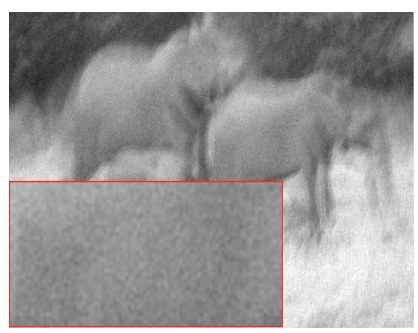

Zoom-in blurred image.

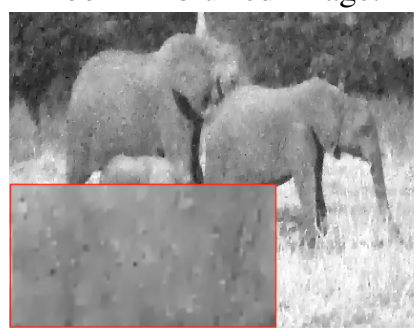

Result of GISA [19].

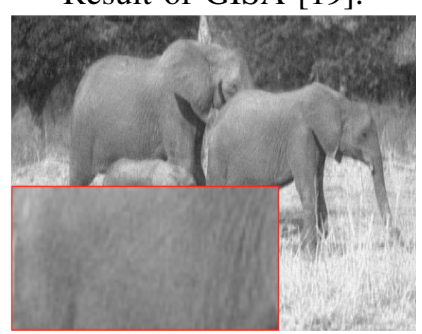

Proposed method.

Figure 4: Performance of the various methods on the Elephant image.

[4] S. H. Chan, R. Khoshabeh, K. B. Gibson, P. E. Gill, and T. Q. Nguyen, "An augmented lagrangian method for total variation video restoration," Image Processing, IEEE Transactions on, vol. 20, no. 11, pp. 30973111, 2011.

[5] J.-L. Starck, F. Murtagh, and J. Fadili, "Sparse image and signal processing: Wavelets, curvelets, morphological diversity," 2011.

[6] J. M. Bioucas-Dias and M. A. Figueiredo, "A new TwIST: two-step iterative shrinkage/thresholding algorithms for image restoration," IEEE Transactions on Image processing, vol. 16, no. 12, pp. 2992-3004, 2007.

[7] "A cross-validation framework for solving image restoration problems,"

Journal of Visual Communication and Image Representation, vol. 3, no. 4, pp. $433-445,1992$.

[8] G. Landi, "A discrete 1-curve for the regularization of ill-posed inverse problems," 2012.

[9] P. C. Hansen, J. G. Nagy, and D. P. O'leary, Deblurring images: matrices, spectra, and filtering. Siam, 2006, vol. 3.

[10] "Synthetic boundary conditions for image deblurring," Linear Algebra and its Applications, vol. 434, no. 11, pp. 2244 - 2268, 2011.

[11] T. Ballal and T. Y. Al-Naffouri, "Improved linear least squares estimation using bounded data uncertainty," in 2015 IEEE International Conference on Acoustics, Speech and Signal Processing (ICASSP). IEEE, 2015, pp. 3427-3431.

[12] M. Suliman, T. Ballal, A. Kammoun, and T. Y. Al-Naffouri, "Constrained perturbation regularization approach for signal estimation using random matrix theory," IEEE Signal Processing Letters, vol. 23, no. 12, pp. 1727-1731, Dec 2016.

[13] A. Alanazi, T. Ballal, M. Masood, and T. Al-Naffouri, "Supplementary material for: Image deblurring using a perturbationbased regularization approach," 2017. [Online]. Available: https://sites.google.com/a/kaust.edu.sa/image-deblurring

[14] C. J. Zarowski, An introduction to numerical analysis for electrical and computer engineers. John Wiley \& Sons, 2004.

[15] B. Efron and R. J. Tibshirani, An introduction to the bootstrap. CRC press, 1994

[16] C. C. Paige and M. A. Saunders, "Lsqr: An algorithm for sparse linear equations and sparse least squares," ACM transactions on mathematical software, vol. 8, no. 1, pp. 43-71, 1982.

[17] "Bayesian non-parametric gradient histogram estimation for textureenhanced image deblurring," Neurocomputing, vol. 197, pp. 95 - 112, 2016.

[18] W. Zuo, D. Meng, L. Zhang, X. Feng, and D. Zhang, "A generalized iterated shrinkage algorithm for non-convex sparse coding," in 2013 IEEE International Conference on Computer Vision, Dec 2013, pp. 217 224.

[19] A. Kheradmand and P. Milanfar, "A general framework for regularized, similarity-based image restoration," IEEE Transactions on Image Processing, vol. 23, no. 12, pp. 5136-5151, Dec 2014.

[20] A. C. Bovik, Handbook of image and video processing. Academic press, 2010.

[21] Z. Wang, A. C. Bovik, H. R. Sheikh, and E. P. Simoncelli, "Image quality assessment: from error visibility to structural similarity," Image Processing, IEEE Transactions on, vol. 13, no. 4, pp. 600-612, 2004. 